\title{
Persistence of Cognitive Impairment after Resolution of Overt Hepatic Encephalopathy
}

\author{
Jasmohan S Bajaj, MD ${ }^{1,4}$, Christine M Schubert, PhD $^{2}$, Douglas M Heuman, $\mathbf{M D}^{1}$, James B \\ Wade, $\mathrm{PhD}^{3}$, Douglas $\mathbf{P}$ Gibson, $\mathrm{PhD}^{3}$, Allyne Topaz, BS ${ }^{1}$, Kia Saeian, $\mathbf{M D}^{4}$, Muhammad \\ Hafeezullah, MBBS ${ }^{4}$, Debulon E Bell, RN ${ }^{1}$, Richard K Sterling, MD ${ }^{1}$, R Todd Stravitz, MD ${ }^{1}$, \\ Velimir Luketic, MD ${ }^{1}$, Melanie B White, BSN ${ }^{1}$, and Arun J Sanyal, MD ${ }^{1}$ \\ 1 Division of Gastroenterology, Hepatology and Nutrition, Virginia Commonwealth University and \\ McGuire VA Medical Center, Richmond, VA \\ ${ }^{2}$ Biostatistics, Virginia Commonwealth University and McGuire VA Medical Center, Richmond, VA \\ ${ }^{3}$ Psychology, Virginia Commonwealth University and McGuire VA Medical Center, Richmond, VA \\ ${ }^{4}$ Division of Gastroenterology and Hepatology, Medical College of Wisconsin, Milwaukee, WI
}

\begin{abstract}
Background \& Aims-In patients with cirrhosis, hepatic encephalopathy (HE) has acute but reversible as well as chronic components. We investigated the extent of residual cognitive impairment following clinical resolution of overt $\mathrm{HE}$ (OHE).
\end{abstract}

Methods-Cognitive function of cirrhotic patients was evaluated using psychometric tests (digit symbol [DS], block design [BD], and number connection [NCT-A\&B]) and the inhibitory control test (ICT). Improvement (reduction) in ICT lures and 1st minus 2 nd halves $\left(\Delta \mathrm{L}_{1-2}\right)$ were used to determine learning of response inhibition. Two cross-sectional studies ( $\mathrm{A}$ and $\mathrm{B}$ ) compared data from stable cirrhotics, with or without prior OHE. We then prospectively assessed cognitive performance, before and after the first episode of OHE.

Results-In study A, (226 cirrhotic patients) 54 had experienced OHE, 120 had minimal HE and 52 with no minimal HE. Despite normal mental status on lactulose after OHE, cirrhotics were cognitively impaired, based on results from all tests. Learning of response inhibition $\left(\Delta \mathrm{L}_{1-2} \geq 1\right)$, was evident in patients with minimal HE and no minimal HE, but was lost after OHE. In study B (50 additional patients who developed $\geq 1$ documented OHE episode during follow-up), the number of OHE hospitalizations correlated with severity of residual impairment, indicated by ICT lures $(\mathrm{r}=0.5$, $\mathrm{P}=0.0001)$, DST $(\mathrm{r}=-0.39, \mathrm{P}=0.002)$ and NCT-B $(\mathrm{r}=0.33, \mathrm{P}=0.04)$. In the prospective study (59 cirrhotics without OHE), 15 developed OHE; ICT lure response worsened significantly after OHE

Correspondence: Jasmohan S Bajaj, MD, MS, Division of Gastroenterology, Hepatology and Nutrition, Virginia Commonwealth University and McGuire VA Medical Center, 1201 Broad Rock Boulevard, Richmond VA 23249, Telephone (804) 675 5802, Fax: (804) 675 5816, jsbajaj@vcu.edu.

Author Involvement: JS Bajaj was involved in all aspects of the study. C Schubert, D Heuman, J Wade and AJ Sanyal were involved in the analysis and interpretation of data, drafting of the manuscript and providing critical revisions to the paper; M Hafeezullah, D Gibson, R Sterling, V Luketic, RT Stravitz, A Topaz, D Bell, M White and K Saeian were involved in data collection

Disclosures: Dr Bajaj is a consultant for and receives independent grant support from Salix and Ocera. Dr Sanyal is a consultant for Salix Pharmaceuticals.

Publisher's Disclaimer: This is a PDF file of an unedited manuscript that has been accepted for publication. As a service to our customers we are providing this early version of the manuscript. The manuscript will undergo copyediting, typesetting, and review of the resulting proof before it is published in its final citable form. Please note that during the production process errors may be discovered which could affect the content, and all legal disclaimers that apply to the journal pertain. 
(12 before vs.18 after, $\mathrm{P}=0.0003$ ) and learning of response inhibition was lost. The 44 patients who did not experience OHE did not have deteriorations in cognitive function in serial testing.

Conclusion-In cirrhosis, episodes of OHE are associated with persistent and cumulative deficits in working memory, response inhibition, and learning.

\section{Keywords}

cirrhosis; portal hypertension; cognition; complications; metabolic brain diseases

\section{Introduction}

Minimal and overt hepatic encephalopathy are constituents of the spectrum of neuro-cognitive impairment in cirrhosis (SONIC) ${ }^{1}$. Minimal hepatic encephalopathy (HE) is defined by impaired performance on psychometric or neurophysiologic testing, despite normal mental status 2. In minimal HE, therapy with agents such as lactulose can completely correct the cognitive abnormalities3. Patients with minimal HE are at increased risk of developing overt $\mathrm{HE}$, characterized by a rostro-caudal progression of symptoms from deficits in attentiveness that may progress to lethargy, asterixis, disorientation, agitation, stupor and coma. While mental status changes in overt HE improve after treatment, there is some evidence that the metabolic insult associated with overt HE may lead to chronic neurological injury that is not readily reversible4. Structural "dementia-like" features have been reported in patients with prior overt HE episodes, accompanied by pathological evidence of neuronal death. Patients with prior overt $\mathrm{HE}$ also have a higher risk of persistent neurological impairment after liver transplantation $4^{-7}$. However, it is unclear whether cognitive deficits persist after resolution of mental status changes in patients with overt HE.

In the current study, we have applied a battery of tests of cognitive function to patients with cirrhosis with and without a prior history of overt HE. We find that with the advent of overt $\mathrm{HE}$, cognitive function is persistently impaired, despite normalization of mental status on lactulose therapy. In particular, learning of response inhibition, as measured using a novel application of the inhibitory control test, is lost in most individuals following the first episode of overt HE. The severity of the persistent impairment increases with the number of episodes of overt HE. These findings are evidence that episodes of overt $\mathrm{HE}$ in cirrhosis lead to neurological injury that is chronic, cumulative and not readily reversible.

\section{Methods}

Subjects for this study included patients with an established diagnosis of hepatic cirrhosis, based on histology or clinical criteria (physical exam, laboratory and imaging findings), who were seen and evaluated in our outpatient clinics in the Medical College of Wisconsin, Virginia Commonwealth University Medical Center and McGuire VA Medical Center. Patients with prior overt $\mathrm{HE}$ were included only if they were receiving appropriate treatment with lactulose and/or rifaximin, were adherent on medications, and had a normal mental status (defined as a mini-mental status examination score $>25$ ). All subjects gave informed consent. Demographic data, etiology, MELD (model for end-stage liver disease) score and details of complications of cirrhosis were collected. If patient had a history of overt HE, the time of first overt HE diagnosis, number of episodes that required hospitalization or emergency room visits, details of therapy and adherence to therapy were investigated and recorded. Patients receiving psychotropic or sedative medications were excluded, as were individuals with known chronic neurological conditions (cerebrovascular accidents, dementia, Parkinson's disease, etc.).

Patients who were non-adherent to therapy, actively abusing alcohol or illicit drugs, or without command of the English language also were excluded. 
The study was divided into cross-sectional and prospective arms. In the cross-sectional arm, part A, we enrolled 226 patients with hepatic cirrhosis evaluated at the Medical College of Wisconsin and Virginia Commonwealth University. These patients were categorized according to whether or not they had previously experienced episodes of overt HE. Based on a standard cognitive battery, patients without prior overt $\mathrm{HE}$ were further characterized as having minimal HE or no minimal HE (normal). In the cross-sectional arm, part B, we studied a separate group of 50 patients with cirrhosis who had been followed longitudinally in our clinics and who had experienced one or more well documented episodes of overt HE. In these patients, severity of cognitive impairment was analyzed with respect to the number, severity and duration of prior overt HE episodes.

In the third, prospective arm, we included 59 cirrhotic patients without prior overt $\mathrm{HE}$ at time of study entry that underwent cognitive testing at least twice between 7/1/07 and 10/1/09 at the Medical College of Wisconsin, Virginia Commonwealth University Medical Center and McGuire VA Medical Center. Of these individuals, 15 experienced a first episode of overt HE during follow-up, while 44 remained free of it The results of cognitive testing were evaluated before and after onset of overt $\mathrm{HE}$, and were compared to results of repeat cognitive testing in patients who remained free of it.

All subjects underwent the following test battery ${ }^{8-10}$

a. Number connection test-A: patients are asked to "join the dots" from 1-25 in a timed manner; it tests for psychomotor speed. Worse performance is indicated by a longer time for completion.

b. Number connection test-B: this involves joining the dots while alternating between numbers and letters and tests for psychomotor speed, set shifting and divided attention. Similar to the number connection test-A, longer time required to complete this indicates worse performance.

c. Digit Symbol test: subjects have to accurately and quickly transcribe nonsense symbols corresponding to numbers looking at a key in a timed manner over 2 minutes; this tests for psychomotor speed, attention and visual memory. The number of correctly transcribed symbols indicates performance, i.e. a low score means poor performance.

d. Block Design test: involves constructing designs made from blocks of increasing complexity in a timed manner. The domains of visuo-motor coordination, visuospatial reasoning, praxis and psychomotor speed are tested. The scores are generated based on accurate construction of designs and a low score again indicates poor performance.

e. Inhibitory control test (ICT): in this computer-based test, patients are shown a series of letters and are asked to respond by pressing a mouse key when an $\mathrm{X}$ is followed by a $\mathrm{Y}$, or a $\mathrm{Y}$ is followed by an $\mathrm{X}$ (alternating presentation, termed targets). Patients are instructed not to respond to $\mathrm{X}$ following $\mathrm{X}$ or $\mathrm{Y}$ following $\mathrm{Y}$ (non-alternating presentation, termed lures11, 12). Cognitive functions required in order to consistently recognize targets include reaction time11, while avoidance of lures requires response inhibition, attention and working memory13. High lure and low target response indicate poor psychometric performance. The ICT is administered as a practice test followed by a series of 6 similar 2-minute runs, separated by breaks to allow the subjects to rest. Runs I-III ( $1^{\text {st }}$ half) are identical to runs IV-VI ( ${ }^{\text {nd }}$ half). Improvement (i.e. reduction) in performance, particularly lure response may serve as a measure of learning. In this study we evaluated a novel ICT parameter, the change 
in lure response between the $1^{\text {st }}$ and the $2^{\text {nd }}$ half, $\left(\Delta \mathrm{L}_{1-2}\right)$, as a measure of learning of response inhibition.

The mini-mental status exam was also administered to all patients with a history of overt HE and only those with a score $>25$ were included for further testing ${ }^{14}$.

Based on prior control subjects' performances and as recommended by the Working Group on Hepatic Encephalopathy, minimal HE was diagnosed if 2 of the 4 tests (number connection tests $\mathrm{A} / \mathrm{B}$, block design and digit symbol tests) were impaired 2 standard deviations beyond normative performance ${ }^{9}$. Cirrhotics without these impairments did not have minimal HE and were termed normal.

\section{Statistical and analytical methods}

In part A of the cross-sectional study, results of the psychometric battery in patients without prior overt $\mathrm{HE}$ (normal and minimal $\mathrm{HE}$ groups) were compared to those of patients with prior HE, using ANOVA and unpaired t-tests. In part B of the cross sectional study, the psychometric test battery was performed in individuals with history of one or more episodes of overt HE. The relationship between psychometric test performance and the number of episodes of overt $\mathrm{HE}$, hospitalizations for overt HE, and the duration of time between the first overt HE episode and the time of psychometric testing were evaluated by Pearson correlation.

In the prospective arm, the psychometric test battery was administered at least twice at intervals of greater than 3 months to individuals who initially were without prior history of overt HE. Changes in psychometric test performance over time were calculated. Patients who were free of overt HE on enrollment and who developed their first overt HE episode during follow-up were compared to patients who remained free of overt HE throughout the test period.

Significance of cognitive change over time in each group was assessed using Student's t test for paired samples, while differences in quantitative change in cognitive performance between groups were assessed using one way ANOVA. All patients with overt HE were outpatients at the time of the psychometric testing. $\Delta \mathrm{L}_{1-2}$ was studied before and after the visits and compared within and between groups who did and did not develop overt HE.

A subgroup analysis of patients with alcoholic versus non-alcoholic etiology of cirrhosis was also performed.

A $p$ value of $p<0.05$ was considered significant and all data are displayed as mean \pm standard deviation unless otherwise noted.

Studies were performed with the approval of the Institutional Review Boards at the Medical College of Wisconsin, Virginia Commonwealth University and McGuire Dept. of Veterans Affairs Medical Center.

\section{Results}

Cross sectional study part A: Of the 226 patients included, 54 had prior overt $\mathrm{HE}$ and $172 \mathrm{did}$ not. Patients without prior overt HE were divided on the basis of the psychometric tests into normal and minimal HE; 52 were normal and the remaining 120 were minimal HE. The demographic and cirrhosis details are displayed in table 1 . The 54 patients with prior overt HE were currently adherent on lactulose (defined by mini-mental score $>25$, corroboration of lactulose use by caregivers and by at least 2 daily bowel movements) were studied. These patients had first experienced overt HE a mean of $12 \pm 5$ months prior to this study. Since this was a cross-sectional report, the details of their overt HE hospitalization and precipitating factors were not adequately reflected in their retrospective records, necessitating the inclusion 
of the second overt HE group (cross-sectional study part B) whose episodes had been documented.

Cognitive testing: Since this was the basis of division, patients with minimal HE were significantly worse on cognitive testing than the normal cirrhotics. On all psychometric tests, patients with overt HE performed significantly worse than normal cirrhotics despite being on adequate therapy. There was no significant difference between patients with an alcoholic etiology of cirrhosis compared to those without alcoholic liver disease. When the psychometric performance between minimal and overt HE was compared, the digit symbol test was significantly more impaired in overt $\mathrm{HE}$ and there was a learning deficit demonstrated in the overt HE patients with respect to lures (Table 1). Normal cirrhotics, as expected had significant reduction (improvement) in their lure response when the $1^{\text {st }}$ half was compared to the $2^{\text {nd }}$ half $(3.4 \pm 2.1$ vs. $2.1 \pm 2, p=0.0001)$. Interestingly minimal HE patients also exhibited a significant learning when the lures of the $1^{\text {st }}$ half were compared to the $2^{\text {nd }}$ half of the ICT $(7.2 \pm 3.8$ vs. $5.7 \pm 4, p=0.0001)$. In sharp contrast, patients with overt HE did not have any significant change when the $1^{\text {st }}$ half was compared to the $2^{\text {nd }}$ half $(6.8 \pm 4.4$ vs. $6.3 \pm 4.8, p=0.24)$, indicating lack of learning (Figure 1).

Cross-sectional study part B; correlation of the additional overt HE group's cognitive performance with number and duration of overt HE episodes: In the fifty additional patients, detailed information on the overt HE episodes was available. The mean age was $56 \pm 6$ years and 39 were men. Of the 50, 38 had hepatitis C, 7 had alcoholic liver disease, 4 had both and 1 had cryptogenic cirrhosis. The mean follow up duration was $13 \pm 12$ months. There were a median of 2 episodes of overt HE (range 1-13) during this period with a median of 1 hospitalization for overt HE (range, 1-7). The precipitating factors for the first hospitalization were infections in 18 (cellulitis in 9, spontaneous bacterial peritonitis in 5 and respiratory infection in 4), transjugular intra-hepatic porto-systemic shunting (TIPS) placement in 10, medication use in 7 patients (narcotics in 5 and zolpidem in 3), post-surgical in 2 and spontaneous in the rest. Overall there were 33 patients with more than 1 admission for overt HE. In these patients, the reason for recurrence was most often non-adherence with lactulose $(n=14)$, infections $(n=7)$ and spontaneous in the rest. At the time of psychometric testing, of the 50, 11 were only on rifaximin, 7 were on both rifaximin and lactulose and the rest 32 were only on lactulose. Rifaximin therapy was only initiated if the patient failed or was non-adherent on lactulose. In between episodes, the patients' mental status was normal and abdominal imaging did not reveal any large shunts in those with recurrent HE episodes.

Scores on tests were number connection test-A $48 \pm 22$ seconds, number connection test-B 149 \pm 87 seconds, digit symbol test raw score $41 \pm 13$, block design test raw score $26 \pm 15$, ICT lures $15 \pm 9$ and ICT targets (\% correct) were $89 \pm 12 \%$. Similar to cross-sectional B, these scores were not statistically different between alcoholic and non-alcoholic patients.

Psychometric scores were highly correlated with the number of episodes of and admissions for overt HE and the time from first overt HE episode to the testing (Table 2). This was especially true for ICT lures, ICT targets, digit symbol and number connection test-B which were significantly correlated with number of admissions for overt HE, number of overt HE episodes and duration from the $1^{\text {st }}$ episode of overt HE until testing. Interestingly number connection test-A and block design tests were not affected by either the number of hospitalizations or overt HE episodes or the duration of overt HE.

\section{Prospective study}

These patients were enrolled to study the natural history of minimal hepatic encephalopathy. A total of 79 patients were enrolled and underwent their first psychometric evaluation. Fifteen did not return for the second scheduled visit because of withdrawn consent or logistical/ 
transport issues, 3 died due to non-hepatic causes and 2 patients received a liver transplant. The remaining 59 were followed and re-tested.

\section{Change before and after development of overt HE}

A total of 15 patients (age $54 \pm 8$ years, 12 men, 11 with chronic hepatitis C, 2 with alcoholic liver disease and 2 with cryptogenic cirrhosis) developed overt HE during the follow-up period, 8 of them after TIPS placement, 5 spontaneously and 2 after an infection. Five patients ( 3 postTIPS and 2 infection-related overt HE) required hospitalization. The rest were diagnosed and treated with lactulose as outpatients and none required rifaximin therapy. The repeat testing was performed $36 \pm 25$ days after the overt HE episode when the patients had been treated with lactulose. Overall the time between the first and the second testing (after the overt HE episode had been treated) was $4 \pm 2$ months.

The mean mini-mental status score post-therapy was $27 \pm 2$ and all patients scored at least 25 on this examination. All patients were adherent on lactulose by history and by corroboration from their relatives.

The psychometric tests did not change or improve after development of overt HE compared to their pre-overt HE baseline despite therapy; apart from an increase in the total ICT lures (Table 2). The pattern of change that was seen in overt HE patients in the cross-sectional arm was again seen in prospective study. Similar to the cross-sectional data, patients prior to overt HE development had a significant learning effect i.e. lures in the first half were much higher than in the $2^{\text {nd }}$ half $(7.5 \pm 5$ vs $4 \pm 4.3$, p=0.0001, Table 3$)$. After development of overt HE and despite adequate therapy this learning capability disappeared and $\Delta \mathrm{L}_{1-2}$ reduced significantly. This was demonstrated by the similar response to lures in the $1^{\text {st }}$ half compared to the $2^{\text {nd }}$ half after they developed overt HE ( $8.7 \pm 5$ vs. $8.1 \pm 5, \mathrm{p}=0.69$, Figure 2$)$. Also corroborating this was no significant difference in lures in the $1^{\text {st }}$ half before or after overt HE development $(\mathrm{p}=0.12)$ but there was a significantly higher lure rate in the $2^{\text {nd }}$ half after overt HE development compared to pre-overt HE testing $(\mathrm{p}=0.012)$. Fourteen of $15(93 \%)$ reduced their lure response from the $1^{\text {st }}$ half to the $2^{\text {nd }}$ half before overt HE compared to only 4 of $15(26 \%, p=0.0001$ Figure $2 \mathrm{~A}$ and $\mathrm{B}$ ).

There was no significant difference in the proportion of patients with alcoholic liver disease who worsened their performance ( 2 of 2 ) compared to the ones who did not have alcoholic liver disease ( 12 of $13, \mathrm{p}=0.9$ ) after overt HE development.

\section{Change in psychometric tests in cirrhotic patients who did not develop overt HE}

There were 44 patients who were tested twice $4.2 \pm 2$ months apart while they remained free of overt HE. These patients were similar in age to the above group and had a mean age of $56 \pm 5$ years. There were 38 men and the leading etiology of cirrhosis was chronic hepatitis $\mathrm{C}$ infection $(n=30)$, the rest had alcoholic liver disease $(n=7)$ and cryptogenic cirrhosis or non-alcoholic steato-hepatitis $(n=7)$. These patients did not have any episodes of overt HE, infections, variceal bleeding, changes in mental status, initiation of psychotropic medications. TIPS placement or significant change in MELD score in between the 2 visits. There were no differences in any of the psychometric tests between these 2 visits in any of the patients. The learning effect on lures was maintained in both visits (i.e. number of lures in the $1^{\text {st }}$ half was significantly higher than the $2^{\text {nd }}$ half, Table 4). This indicates that in patients with no change in clinical status, their psychometric performance remains stable in minimal HE over time. Comparisons between those with alcoholic versus non-alcoholics regarding all psychometric tests between the 2 visits were not significant. 
This is in contrast with the patients who develop overt HE, in which there are changes in learning capacity and working memory even after 1 episode of overt HE despite complete resolution of mental status abnormalities.

Comparison of change in psychometric performance between the two prospectively studied groups: There were no significant changes in any psychometric test apart from ICT lures between these 2 groups. However, ICT lures increased in those who developed overt HE compared to those who did not; the difference in the total lures was significantly higher in those with overt $\mathrm{HE}$ (Table 5). $\Delta \mathrm{L}_{1-2}$ was also significantly lower after overt $\mathrm{HE}$ development compared to before overt $\mathrm{HE}$ while $\Delta \mathrm{L}_{1-2}$ remained unchanged between visits for patients who did not develop overt HE. Also when the difference of $\Delta \mathrm{L}_{1-2}$ from each visits were compared, there was a significant change in the group that developed overt HE compared to the overt HEfree group.

\section{Discussion}

The spectrum of neuro-cognitive impairment in cirrhosis (SONIC) forms a continuum that spans the range from normal cognitive function to minimal $\mathrm{HE}$ to overt $\mathrm{HE}^{1}, 13$. Both minimal and overt hepatic encephalopathy are generally considered to be fully reversible with treatment15. However, a variety of lines of evidence suggest that the metabolic derangement caused by ammonia and other neurotoxins in cirrhosis may result in neurological injury that can be persistent, and possibly permanent5, 16-19. In autopsy series, brains of patients dying in coma associated with overt HE exhibited definite structural abnormalities ${ }^{5-7,20}$. There may also be residual neurological findings on physical examination in overt HE patients ${ }^{21,} 22$. Patients with history of overt HE have an increased likelihood of persistent neuro-cognitive dysfunction after liver transplant compared to those without prior overt $\mathrm{HE}^{4}$. Children with chronically elevated ammonia due to hereditary urea cycle disorders can develop irreversible central nervous system damage ${ }^{23}$. The pathogenesis of these durable changes that may persist despite resolution of the mental status are not fully understood.

In the current study, cognitive impairment in patients with history of overt HE was more severe than in patients without prior overt HE, and severity of impairment increased with the number of previous episodes of overt HE. In patients followed prospectively, we found that a single episode of overt $\mathrm{HE}$ was accompanied by acquisition of a defect in learning of response inhibition, as measured by the ICT. In patients with multiple episodes of overt HE, we noted additional defects in reaction times, set shifting, divided attention, response inhibition and working memory as measured by digit symbol test, number connection test-B and ICT lures and targets. These tests measure integrity of reaction times, psychomotor speed, set shifting and divided attention. Poor performance on these cognitive measures was not merely a reflection of generalized psychomotor slowing, since we found no relationship between overt $\mathrm{HE}$ and performance on number connection test-A (a measure of psychomotor speed) or the block design test (a measure of visuo-motor coordination). Our findings indicate that there is cumulative worsening of performance in basic domains of cognitive function following recurrent episodes of overt HE. Working memory and frontal regulatory circuit impairment, measured by ICT lure response, occurs early, while loss of other functions such as set shifting, sustained attention, and visual memory indicates a more advanced stage in the SONIC. This is in concordance with the rostro-caudal progression of deficits that has been demonstrated in metabolic encephalopathies through animal studies ${ }^{24}$. Our data also suggest that decline in working memory and the integrity of frontal regulatory circuits, followed by other forms of cognitive dysfunction may define the neurobehavioral decline in overt HE. It could be argued that the worse cognitive impairment in overt HE may be due to factors such as impaired volition or neuro-muscular difficulties. However, this is unlikely since ICT target response, which measures response time, was intact in all patients with overt HE. Therefore, these findings 
point to a loss of "cognitive reserve" in overt HE patients and may make them increasingly vulnerable to neurobehavioral decline with future changes in brain homeostasis.

The ICT is a dynamic and fast-paced task that interrogates working memory, learning capacity and response inhibition ${ }^{12}$. In particular, it depends upon proper function of the cortical association areas necessary for orienting attentional resources and analysis of critical features for identifying salient stimuli ${ }^{12}$. The prefrontal cortex is important for screening distracters and shifting/dividing attention in a task appropriate manner. Further, the prefrontal cortex and angular gyrus are needed for the allocation of attentional resources to achieve target goals in organizing thought/behavior ${ }^{25,26}$. Successful lure inhibition in the ICT requires the subject to critically evaluate each stimulus presented in a sequence which conforms to the n-back task which requires the continuous process of building, maintaining, updating, and releasing arbitrary bindings between items in temporal order positions 27,28 . The top-down control of posterior parietal cortex via dorso-lateral prefrontal cortex modulation has been proposed to underlie optimal working memory performance during the n-back task. As task difficulty increases, prefrontal excitatory input enhances parietal region activity, thereby increasing memory capacity. Our findings in this study are consistent with those of other studies that have shown impaired activation of the pre-frontal cortex in patients with minimal and early overt $\mathrm{HE}^{29,} 30$.

The mechanisms behind the lack of reversibility of the neuro-cognitive status despite resolution of mental status changes are unclear. It was also interesting to note that despite therapy with ammonia-lowering strategies, these deficits persisted. This brings up the possibility that toxins other than ammonia, such as accumulation of manganese, inflammatory cytokines or mercaptans may be implicated in this persistence ${ }^{18}, 19,31$. There is also conflicting evidence regarding the molecular mechanisms of these persistent cognitive changes in overt HE.

Autopsy and animal studies have implicated changes in neurotransmitter systems such as neurosteroids, mono-amines and opioids in the hippocampus and frontal cortex in metabolic encephalopathies such as overt $\mathrm{HE}^{16,17,32-34}$. Kril et al also suggested that thalamic neuronal cell loss in overt HE in patients may contribute to persistent changes in cognition in alcoholic cirrhotics $^{20}$; however there was no specific difference in the cognitive impairment between alcoholics and non-alcoholics in our patient population. Therefore further studies are required to elucidate the underlying mechanisms behind the complex cognitive changes in cirrhosis.

The finding that episodes of overt HE can lead to persistent cognitive impairment may have important implications in assigning priority for liver transplantation. Previous studies have found that overt HE adversely affects pre-transplant survival independently of the MELD score, and patients with pre-transplant overt HE have a higher incidence of post transplant neurological complications ${ }^{4,8}$. If brain damage associated with episodes of overt HE is prolonged or permanent, then earlier transplantation may be warranted in patients whose encephalopathy is difficult to control with conventional therapies.

In conclusion, this study demonstrates that there are residual effects on cognitive function, especially executive functions that result in learning impairment and working memory problems in patients with overt HE, even after their first episode despite adequate therapy and the attainment of normal mental status. This psychometric performance deterioration continues and expands to the more basic cognitive domains of psychomotor speed, set shifting and divided attention with increasing numbers of episodes and hospitalizations for overt HE. Further prospective studies are needed to determine the long term cognitive consequences of overt $\mathrm{HE}$ and benefits of expedited transplantation in patients with recurrent overt HE. 


\section{Acknowledgments}

Grant Support: This was supported in part by the American College of Gastroenterology Junior Faculty Development Award, and by clinical research center grants MO1-RR00065 and MO1-RR00058, NCRR, NIH (awarded to JSB).

\section{Abbreviations}

SONIC spectrum of neuro-cognitive impairment in cirrhosis

MELD model for end-stage liver disease score

HE hepatic encephalopathy

ICT inhibitory control test

$\Delta \mathrm{L}_{1-2} \quad$ Lures on the first half of ICT minus those in the second half

\section{References}

1. Bajaj JS, Wade JB, Sanyal AJ. Spectrum of neurocognitive impairment in cirrhosis: Implications for the assessment of hepatic encephalopathy. Hepatology 2009;50:2014-21. [PubMed: 19787808]

2. Ortiz M, Jacas C, Cordoba J. Minimal hepatic encephalopathy: diagnosis, clinical significance and recommendations. J Hepatol 2005;42 (Suppl):S45-53. [PubMed: 15777572]

3. Prasad S, Dhiman RK, Duseja A, Chawla YK, Sharma A, Agarwal R. Lactulose improves cognitive functions and health-related quality of life in patients with cirrhosis who have minimal hepatic encephalopathy. Hepatology 2007;45:549-559. [PubMed: 17326150]

4. Sotil EU, Gottstein J, Ayala E, Randolph C, Blei AT. Impact of preoperative overt hepatic encephalopathy on neurocognitive function after liver transplantation. Liver Transpl 2009;15:184-92. [PubMed: 19177446]

5. Butterworth RF, Giguere JF, Michaud J, Lavoie J, Layrargues GP. Ammonia: key factor in the pathogenesis of hepatic encephalopathy. Neurochem Pathol 1987;6:1-12. [PubMed: 3306479]

6. Rose C, Jalan R. Is minimal hepatic encephalopathy completely reversible following liver transplantation? Liver Transpl 2004;10:84-7. [PubMed: 14755783]

7. Butterworth RF. Neuronal cell death in hepatic encephalopathy. Metab Brain Dis 2007;22:309-20. [PubMed: 17851742]

8. Stewart CA, Malinchoc M, Kim WR, Kamath PS. Hepatic encephalopathy as a predictor of survival in patients with end-stage liver disease. Liver Transpl 2007;13:1366-71. [PubMed: 17520742]

9. Ferenci P, Lockwood A, Mullen K, Tarter R, Weissenborn K, Blei AT. Hepatic encephalopathy-definition, nomenclature, diagnosis, and quantification: final report of the working party at the 11th World Congresses of Gastroenterology, Vienna, 1998. Hepatology 2002;35:716-21. [PubMed: 11870389]

10. Weissenborn K, Ennen JC, Schomerus H, Ruckert N, Hecker H. Neuropsychological characterization of hepatic encephalopathy. J Hepatol 2001;34:768-73. [PubMed: 11434627]

11. Bajaj JS, Hafeezullah M, Franco J, Varma RR, Hoffmann RG, Knox JF, Hischke D, Hammeke TA, Pinkerton SD, Saeian K. Inhibitory control test for the diagnosis of minimal hepatic encephalopathy. Gastroenterology 2008;135:1591-1600. e1. [PubMed: 18723018]

12. Garavan H, Ross TJ, Stein EA. Right hemispheric dominance of inhibitory control: an event-related functional MRI study. Proc Natl Acad Sci U S A 1999;96:8301-6. [PubMed: 10393989]

13. Bajaj JS, Saeian K, Schubert CM, Hafeezullah M, Franco J, Varma RR, Gibson DP, Hoffmann RG, Stravitz RT, Heuman DM, Sterling RK, Shiffman M, Topaz A, Boyett S, Bell D, Sanyal AJ. Minimal hepatic encephalopathy is associated with motor vehicle crashes: the reality beyond the driving test. Hepatology 2009;50:1175-83. [PubMed: 19670416]

14. Folstein MF, Folstein SE, McHugh PR. "Mini-mental state". A practical method for grading the cognitive state of patients for the clinician. J Psychiatr Res 1975;12:189-98. [PubMed: 1202204] 
15. Garcia-Tsao G, Lim JK. Management and treatment of patients with cirrhosis and portal hypertension: recommendations from the Department of Veterans Affairs Hepatitis C Resource Center Program and the National Hepatitis C Program. Am J Gastroenterol 2009;104:1802-29. [PubMed: 19455106]

16. Bergeron M, Reader TA, Layrargues GP, Butterworth RF. Monoamines and metabolites in autopsied brain tissue from cirrhotic patients with hepatic encephalopathy. Neurochem Res 1989;14:853-9. [PubMed: 2480534]

17. de Waele JP, Audet RM, Leong DK, Butterworth RF. Portacaval anastomosis induces region-selective alterations of the endogenous opioid system in the rat brain. Hepatology 1996;24:895-901. [PubMed: 8855194]

18. Pomier-Layrargues G, Spahr L, Butterworth RF. Increased manganese concentrations in pallidum of cirrhotic patients. Lancet 1995;345:735. [PubMed: 7885158]

19. Zieve L, Doizaki WM, Zieve J. Synergism between mercaptans and ammonia or fatty acids in the production of coma: a possible role for mercaptans in the pathogenesis of hepatic coma. J Lab Clin Med 1974;83:16-28. [PubMed: 4808653]

20. Kril JJ, Butterworth RF. Diencephalic and cerebellar pathology in alcoholic and nonalcoholic patients with end-stage liver disease. Hepatology 1997;26:837-41. [PubMed: 9328301]

21. Krieger S, Jauss M, Jansen O, Theilmann L, Geissler M, Krieger D. Neuropsychiatric profile and hyperintense globus pallidus on T1-weighted magnetic resonance images in liver cirrhosis. Gastroenterology 1996;111:147-55. [PubMed: 8698193]

22. Mullen KD, Cole M, Foley JM. Neurological deficits in "awake" cirrhotic patients on hepatic encephalopathy treatment: missed metabolic or metal disorder? Gastroenterology 1996;111:256-7. [PubMed: 8698210]

23. Gropman AL, Batshaw ML. Cognitive outcome in urea cycle disorders. Mol Genet Metab 2004;81 (Suppl 1):S58-62. [PubMed: 15050975]

24. Giguere JF, Butterworth RF. Amino acid changes in regions of the CNS in relation to function in experimental portal-systemic encephalopathy. Neurochem Res 1984;9:1309-21. [PubMed: 6150451]

25. Derbyshire SW, Vogt BA, Jones AK. Pain and Stroop interference tasks activate separate processing modules in anterior cingulate cortex. Exp Brain Res 1998;118:52-60. [PubMed: 9547077]

26. Passingham RE. Attention to action. Philos Trans R Soc Lond B Biol Sci 1996;351:1473-9. [PubMed: 8941959]

27. Cohen JD, Perlstein WM, Braver TS, Nystrom LE, Noll DC, Jonides J, Smith EE. Temporal dynamics of brain activation during a working memory task. Nature 1997;386:604-8. [PubMed: 9121583]

28. Friedman NP, Miyake A, Corley RP, Young SE, Defries JC, Hewitt JK. Not all executive functions are related to intelligence. Psychol Sci 2006;17:172-9. [PubMed: 16466426]

29. Zafiris O, Kircheis G, Rood HA, Boers F, Haussinger D, Zilles K. Neural mechanism underlying impaired visual judgement in the dysmetabolic brain: an fMRI study. Neuroimage 2004;22:541-52. [PubMed: 15193582]

30. Zhang LJ, Yang G, Yin J, Liu Y, Qi J. Neural mechanism of cognitive control impairment in patients with hepatic cirrhosis: a functional magnetic resonance imaging study. Acta Radiol 2007;48:57787. [PubMed: 17520437]

31. Shawcross DL, Wright G, Olde Damink SW, Jalan R. Role of ammonia and inflammation in minimal hepatic encephalopathy. Metab Brain Dis 2007;22:125-38. [PubMed: 17260161]

32. Fan P, Lavoie J, Le NL, Szerb JC, Butterworth RF. Neurochemical and electrophysiological studies on the inhibitory effect of ammonium ions on synaptic transmission in slices of rat hippocampus: evidence for a postsynaptic action. Neuroscience 1990;37:327-34. [PubMed: 1966824]

33. Ahboucha S, Layrargues GP, Mamer O, Butterworth RF. Increased brain concentrations of a neuroinhibitory steroid in human hepatic encephalopathy. Ann Neurol 2005;58:169-70. [PubMed: 15984019]

34. Butterworth RF, Le O, Lavoie J, Szerb JC. Effect of portacaval anastomosis on electrically stimulated release of glutamate from rat hippocampal slices. J Neurochem 1991;56:1481-4. [PubMed: 1672881] 


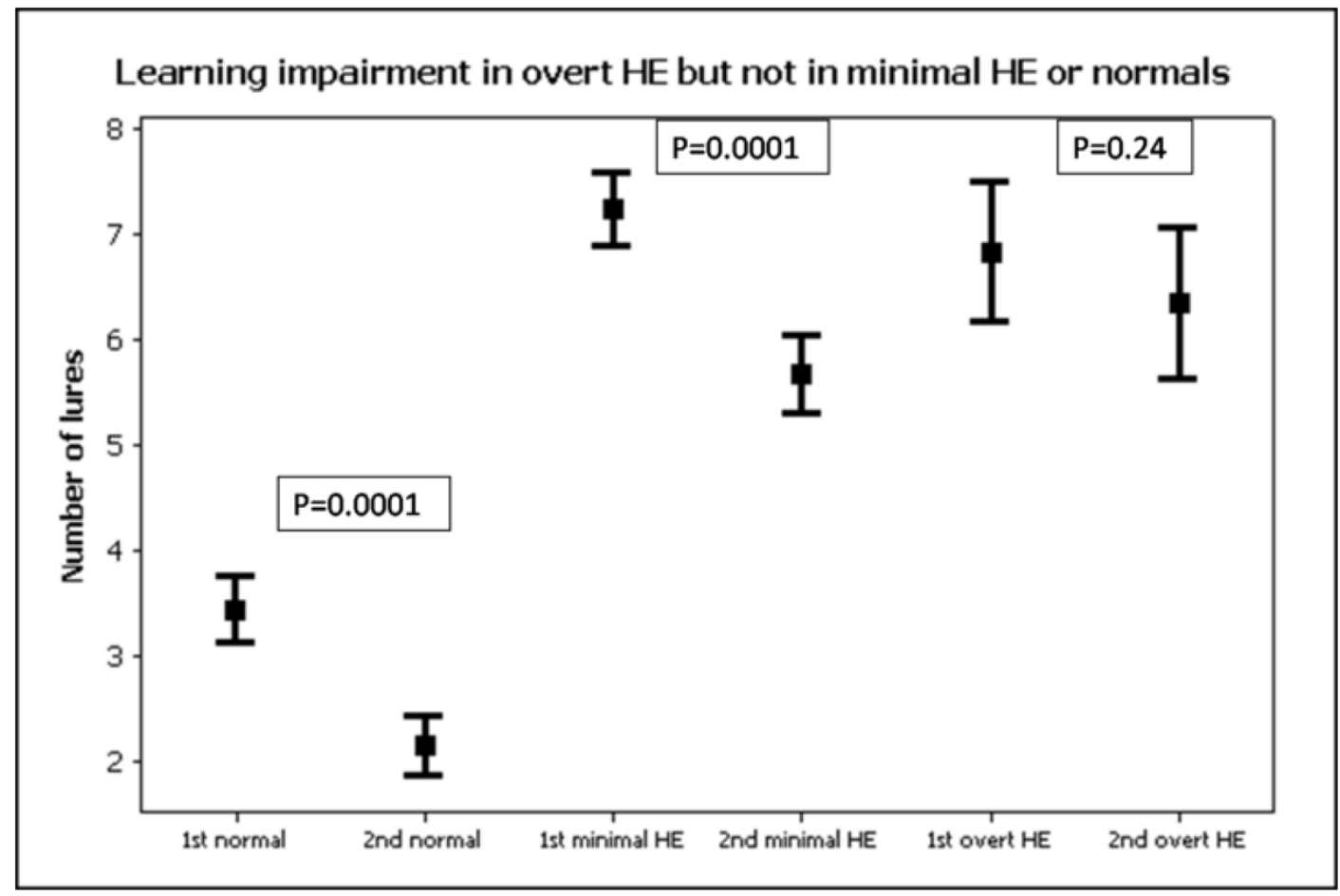

Figure 1. Learning impairment in patients with cirrhosis and overt hepatic encephalopathy Figure 1: The $1^{\text {st }}$ half of the ICT is identical to the $2^{\text {nd }}$ half, therefore learning capability and working memory can be tested using ICT performance. There was a significant learning effect in normal cirrhotics and those with minimal HE but not in overt HE. This indicates a learning impairment in overt HE despite adequate therapy in these patients. A high number of lures

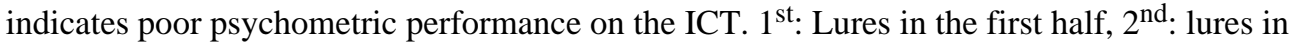
the second half, ICT: inhibitory control test, HE: hepatic encephalopathy, overt HE: recent overt hepatic encephalopathy controlled on lactulose therapy. 
Figure 2A

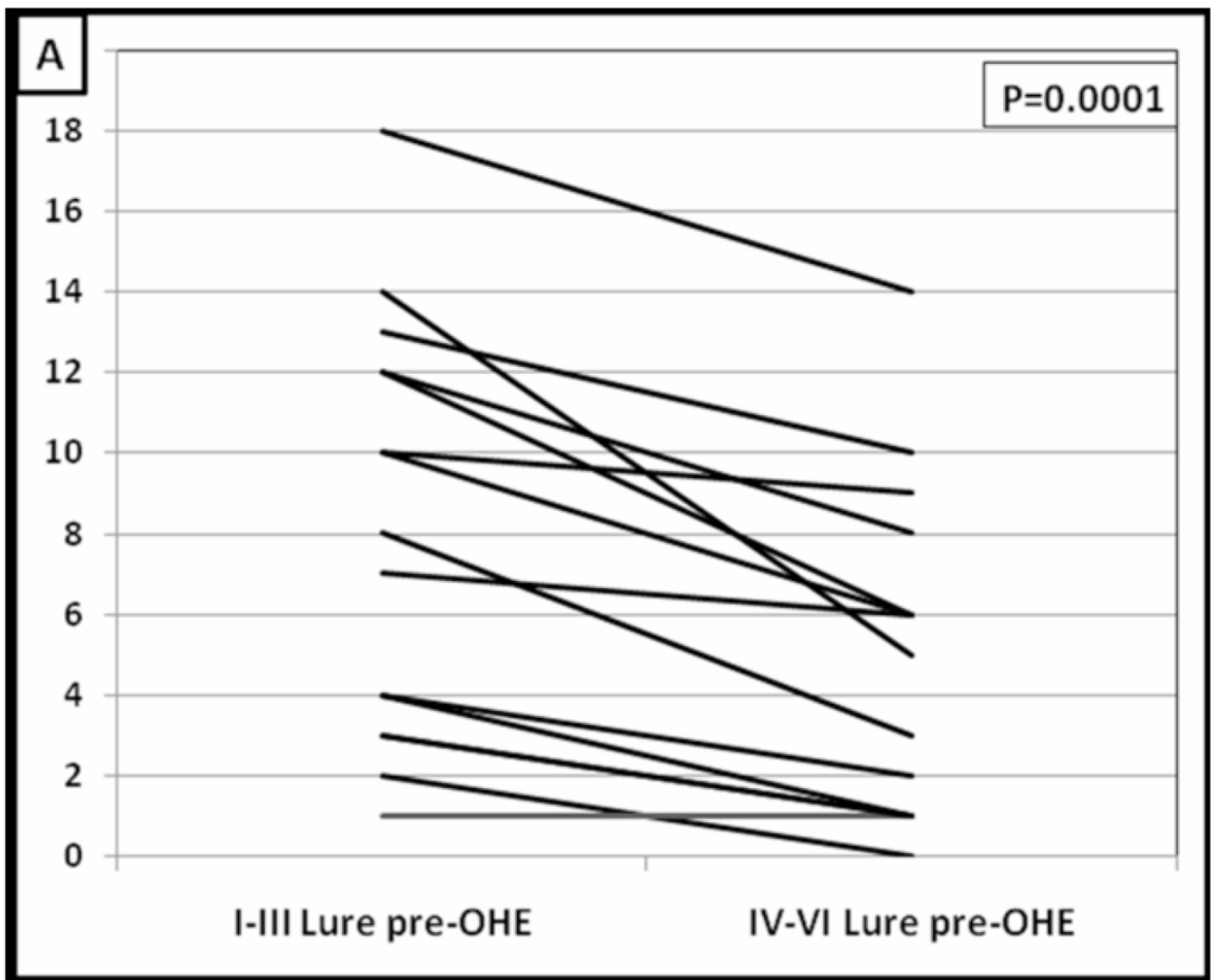


Figure 2B

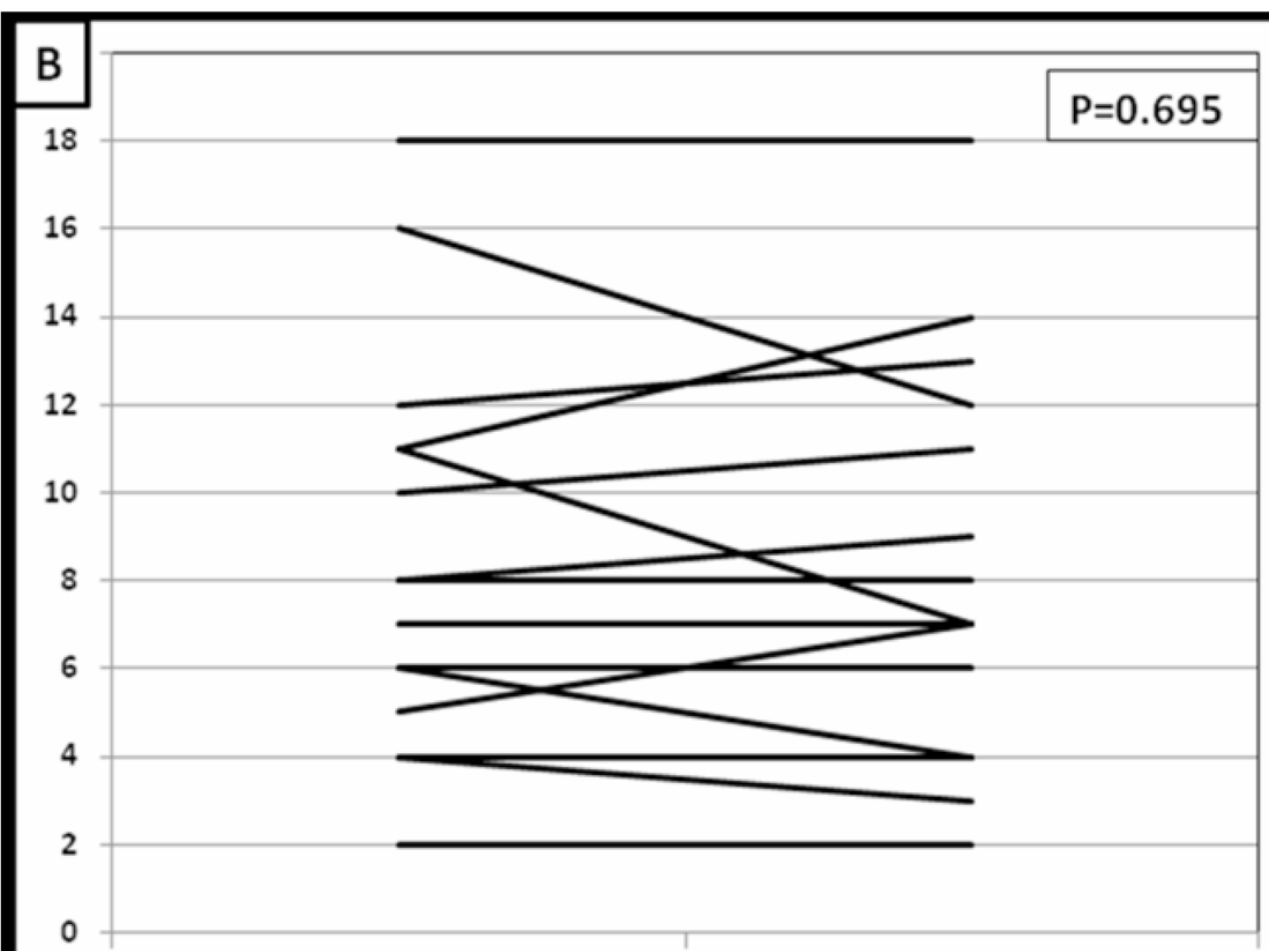

I-III lures post-OHE

IV-VI lures post OHE

Figure 2. Change in lure performance on the ICT in patients before and after the development of overt hepatic encephalopathy

Figure 2A: There was a significant improvement in lures before overt HE development.

Figure 2B: After those patients developed overt HE, this learning effect on lures disappeared. Fourteen of $15(93 \%)$ patients were able to learn the ICT before developing overt HE while only $4(26 \%)$ were able to reduce their lures once they developed overt HE ( $p=0.0001)$. I-III lures: Lures in the $1^{\text {st }}$ half of the ICT, IV-VI lures: Lures in the $2^{\text {nd }}$ half of the ICT, OHE: overt hepatic encephalopathy controlled on lactulose therapy. 


\begin{tabular}{|c|c|c|c|c|c|c|c|c|c|c|c|c|}
\hline & 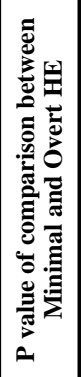 & 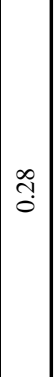 & $\stackrel{7}{\circ}$ & 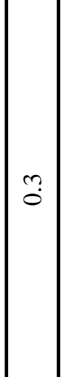 & 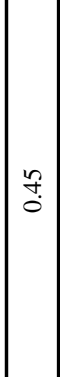 & ปี & તુ & @̊̊ & $\mid \begin{array}{l}0 \\
0 \\
0 \\
0\end{array}$ & $\mid \begin{array}{l}\hat{\imath} \\
\hat{0}\end{array}$ & $\stackrel{\circ}{0}$ & \\
\hline 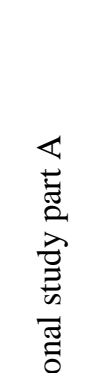 & 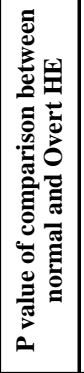 & กุ & $\stackrel{ \pm}{0}$ & $n$ & $\stackrel{\%}{0}$ & 产 & $\mid \begin{array}{l}\overline{\hat{\sigma}} \\
\stackrel{0}{0}\end{array}$ & 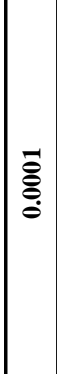 & 㝘 & $\mid \begin{array}{c}\overline{\mathbf{t}} \\
\stackrel{0}{\circ}\end{array}$ & 言 & \\
\hline 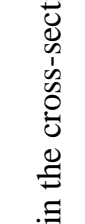 & 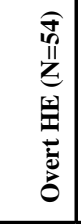 & $\begin{array}{l}\overrightarrow{+} \\
i n \\
n\end{array}$ & $\begin{array}{l}\overrightarrow{+1} \\
\cong\end{array}$ & $\mid \begin{array}{l}\frac{2}{2} \\
\frac{2}{2} \\
\frac{c}{2}\end{array}$ & 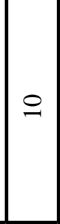 & $\mid \begin{array}{c}c \\
m \\
+1 \\
+ \\
y\end{array}$ & 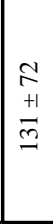 & $\mid \begin{array}{l}\infty \\
+1 \\
+ \\
+ \\
+\end{array}$ & $\begin{array}{l}= \\
+1 \\
++1 \\
0 \\
i\end{array}$ & $\begin{array}{l}a \\
+1 \\
2\end{array}$ & $\begin{array}{l}\stackrel{0}{+1} \\
+1 \\
\sigma\end{array}$ & \\
\hline 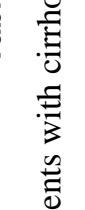 & 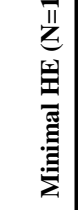 & $\begin{array}{l}-+1 \\
+1 \\
n\end{array}$ & $\begin{array}{l}\sim \\
+1 \\
\pm\end{array}$ & 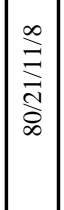 & $a$ & $\begin{array}{l}n \\
n+1 \\
\infty \\
\infty\end{array}$ & $\begin{array}{l}8 \\
0 \\
+1 \\
\cong \\
=\end{array}$ & $\begin{array}{l}m \\
n+1 \\
n \\
n\end{array}$ & $\begin{array}{l}= \\
+1 \\
\vec{\lambda}\end{array}$ & $\begin{array}{l}- \\
+1 \\
-1\end{array}$ & $\begin{array}{l}\sigma \\
+ \\
\alpha \\
\sigma\end{array}$ & \\
\hline 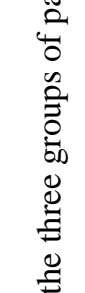 & 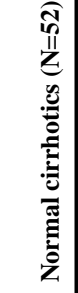 & $\begin{array}{l}\tilde{N} \\
+ \\
\tilde{N}\end{array}$ & $\begin{array}{l}\sim \\
+1 \\
\pm\end{array}$ & 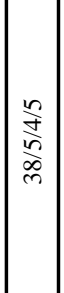 & $\infty$ & 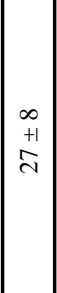 & $\begin{array}{c}F \\
f \\
i n \\
\end{array}$ & $\mid \begin{array}{l}0 \\
2 \\
+1 \\
2\end{array}$ & $\begin{array}{l}\approx \\
+1 \\
m \\
m\end{array}$ & $\begin{array}{l}+ \\
++ \\
i n\end{array}$ & $\begin{array}{c}m \\
+1 \\
\infty\end{array}$ & \\
\hline 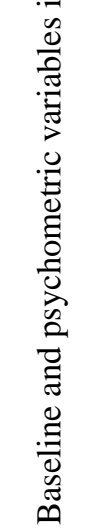 & & 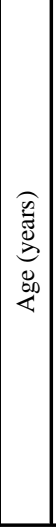 & 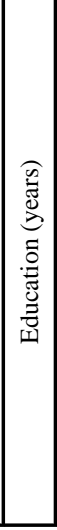 & 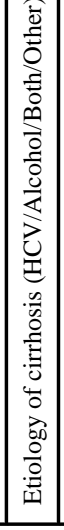 & 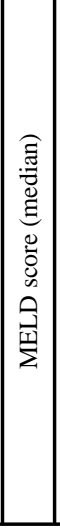 & 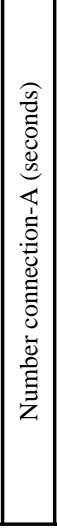 & 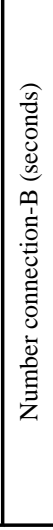 & 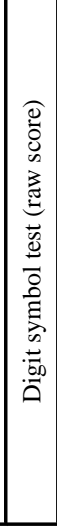 & 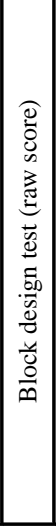 & 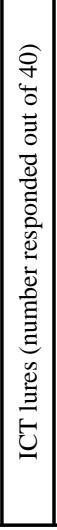 & 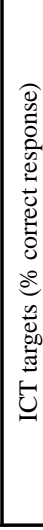 & \\
\hline
\end{tabular}




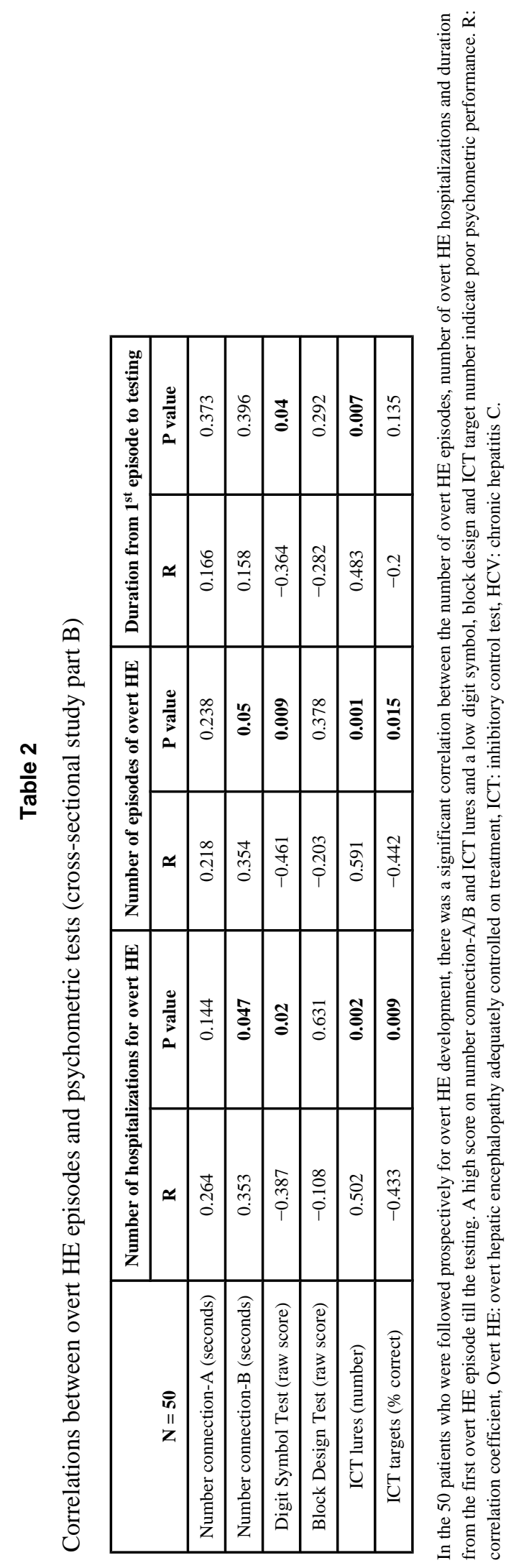

Gastroenterology. Author manuscript; available in PMC 2011 June 1. 
Table 3

Psychometric test results of patients tested before and after development of their first episode of overt HE

\begin{tabular}{|c|c|c|c|}
\hline \multirow{2}{*}{} & \multicolumn{2}{|c|}{ Patients tested before and after development of the first episode of overt HE (n=15) } \\
\cline { 2 - 4 } & Pre-overt HE & Post-overt HE 1 $\mathbf{1}^{\text {st }}$ episode & $\begin{array}{c}\text { Paired t-test p values (before and after the } \\
\text { episode) }\end{array}$ \\
\hline MELD score (median) & 9 & 10 & 0.10 \\
\hline Number connection test-A (seconds) & $40 \pm 12$ & $48 \pm 35$ & 0.33 \\
\hline Number connection test-B (seconds) & $98 \pm 22$ & $142 \pm 98$ & 0.11 \\
\hline Digit Symbol Test (raw score) & $51 \pm 14$ & $47 \pm 15$ & 0.21 \\
\hline Block Design Test (raw score) & $29 \pm 13$ & $33 \pm 20$ & 0.39 \\
\hline ICT targets (\% correct) & $93 \pm 9$ & $93 \pm 11$ & 0.96 \\
\hline ICT lures (number responded to) & $12 \pm 8$ & $18 \pm 10$ & $\mathbf{0 . 0 3}$ \\
\hline ICT lures (1 ${ }^{\text {st }}$ half: runs I-III) & $8 \pm 5$ & $9 \pm 5$ & 0.12 \\
\hline ICT lures (2 ${ }^{\text {nd }}$ half: runs IV-VI) & $4 \pm 4^{*}$ & $8 \pm 5$ & $\mathbf{0 . 0 1 2}$ \\
\hline$\Delta \mathrm{L}_{1-2}$ (lures in the $1^{\text {st }}$ half minus the $2^{\text {nd }}$ half) & 3.1 & 0.3 & $\mathbf{0 . 0 1}$ \\
\hline
\end{tabular}

p $=0.00001$ in the $1^{\text {st }}$ half compared to the $2^{\text {nd }}$ half indicating successful learning.

There was a significant worsening of ICT lures after development of overt HE and the learning effect on the ICT which was present before overt HE disappeared. A high score on number connection-A/B and ICT lures and a low digit symbol, block design and ICT target number indicate poor psychometric performance. MELD: model for end-stage liver disease score, Overt HE: overt hepatic encephalopathy adequately controlled on treatment, ICT: inhibitory control test. 


\section{Table 4}

Results of repeat testing in patients undergoing psychometric evaluation twice without interval development of overt HE

\begin{tabular}{|c|c|c|c|}
\hline \multirow{2}{*}{} & \multicolumn{2}{|c|}{ Patients tested twice without interval development of overt HE $(\mathbf{n}=\mathbf{4 4})$} \\
\cline { 2 - 4 } & Baseline & Follow-up & Paired t-test p value \\
\hline MELD score (median) & 9 & 9 & 0.76 \\
\hline Number connection test-A (seconds) & $39 \pm 15$ & $38 \pm 18$ & 0.75 \\
\hline Number connection test-B (seconds) & $110 \pm 69$ & $105 \pm 62$ & 0.73 \\
\hline Digit Symbol Test (raw score) & $56 \pm 12$ & $57 \pm 20$ & 0.09 \\
\hline Block Design Test (raw score) & $29 \pm 13$ & $33 \pm 20$ & 0.39 \\
\hline ICT targets (\% correct) & $95 \pm 5$ & $94 \pm 9$ & 0.54 \\
\hline ICT lures (number) & $11 \pm 7$ & $11 \pm 5$ & 0.97 \\
\hline ICT lures (1 $1^{\text {st }}$ half: runs I-III) & $7 \pm 3$ & $8 \pm 4$ & 0.23 \\
\hline ICT lures $\left(2^{\text {nd }}\right.$ half: runs IV-VI) & $4 \pm 3^{*}$ & $4 \pm 4^{*}$ & 0.45 \\
\hline$\Delta \mathrm{L}_{1-2}$ (lures in the $1^{\text {st }}$ half minus the $2^{\text {nd }}$ half) & 2.1 & 1.8 & 0.52 \\
\hline
\end{tabular}

p $=0.001$ in the $1^{\text {st }}$ half compared to the $2^{\text {nd }}$ half indicating successful learning.

There was no significant difference in any psychometric test or MELD score in the group that was tested twice without the interval development of overt HE and the learning effect on the ICT was maintained in both visits. A high score on number connection-A/B and ICT lures and a low digit symbol, block design and ICT target number indicate poor psychometric performance. MELD: model for end-stage liver disease score, Overt HE: overt hepatic encephalopathy adequately controlled on treatment, ICT: inhibitory control test. 
Table 5

Comparison of ICT lure performance between patients who developed overt HE and those who remained overt HE-free

\begin{tabular}{|c|c|c|c|}
\hline & Developed overt HE $(\mathbf{n = 1 5})$ & Did not develop overt HE $(\mathbf{n = 4 4})$ & P value between groups \\
\hline Total lures on Visit 1 minus Visit 2 & 5.2 & 0.2 & $\mathbf{0 . 0 0 0 2}$ \\
\hline $1^{\text {st }}$ visit $\Delta \mathrm{L}_{1-2}$ & 3.1 & 2.1 & 0.56 \\
\hline $2^{\text {nd }}$ visit $\Delta \mathrm{L}_{1-2}$ & 0.3 & 1.8 & $\mathbf{0 . 0 0 0 1}$ \\
\hline Change in $\Delta \mathrm{L}_{1-2}$ between visits & 2.8 & 0.3 & $\mathbf{0 . 0 0 0 1}$ \\
\hline
\end{tabular}

There was a significant change over time in total lures in patients who developed their first overt HE episode compared to those who did not.

$\Delta \mathrm{L}_{1-2}$ : Lures in runs I-III ( $1^{\text {st }}$ half) minus lures in runs IV-VI ( $2^{\text {nd }}$ half), a higher number indicates learning; this was similar between groups at baseline. Once they developed overt $\mathrm{HE}, \Delta \mathrm{L}_{1-2}$ declined while it remained stable in those who did not develop overt $\mathrm{HE}$ and the change in $\Delta \mathrm{L}_{1-2}$ i.e. extent of change of leaning ability was also significantly different. Overt HE: overt hepatic encephalopathy adequately controlled on treatment, ICT: inhibitory control test. 